\title{
Equivalence of Three Seemingly Different Phases of Ni-rich Li-ion Battery Cathodes- New Insights Using Combined STEM and EELS Study
}

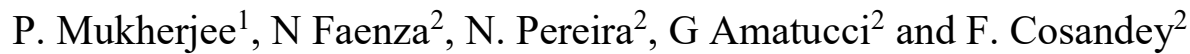 \\ 1. Department of Materials Science \& Engineering, Michigan Technological University, Houghton, MI. \\ 2. Department of Materials Science \& Engineering, Rutgers University, Piscataway, NJ.
}

Ni-rich $\mathrm{LiNi}_{0.8} \mathrm{Co}_{0.15} \mathrm{Al}_{0.05} \mathrm{O}_{2}$, commonly known as $\mathrm{NCA}$, is one of the highest capacity cathode materials currently being used commercially. Although the commercial use of the NCA cathodes is underway, the formation of surface phases after high state of charge prohibits its full theoretical capacity. The general understanding, based on recent literature review, is that a transformation from parent layered to cubic spinel and then finally to a rocksalt phase happens at the cathode surface with increasing charge voltage. In an ideal rocksalt $\mathrm{NiO}$ phase Li transport is very limited as all Li sites are occupied by $\mathrm{Ni}^{+2}$ ions. Experimental reports [1], however, show active Li transport through these cathodes even when charged to $4.75 \mathrm{~V}$ despite the presence of rocksalt phase covering vast area of the cathode surface. This suggests that the complex phases, often containing Li, form at the cathode surface. The real crystallographic and chemical nature of these surface phases would explain Li transport, determine ways to form specific phases with more Li channels, and conditions of their formation. Recently, Ceder's group has calculated stable phases in Li-Ni-O ternary system with various $\mathrm{Li}, \mathrm{Ni}$, and $\mathrm{O}$ concentrations [2]. Experimentally, many of these phases have not yet been discovered. In this work, we are studying the crystallographic and chemical nature of surface phases formed in $\mathrm{LiNiO}_{2}(\mathrm{LNO})$ after 2 weeks of ageing at $4.75 \mathrm{~V}$. We have chosen LNO as a model system for this work instead of NCA as the phases found here can be directly correlated with the theory and the presence of Al in NCA makes quantitative analysis difficult. For the present work, high resolution STEM imaging and EELS chemical measurements have been performed from the same area.

The crystal structures of LNO compounds with varying compositions were observed in an aberration corrected FEI Titan microscope (TEAM I at Lawrence Berkeley National Laboratory). The spatial resolution of the STEM system was $0.07 \mathrm{~nm}$. EELS chemical analysis was performed in the same instrument using a GATAN Enfina EELS spectrometer with an energy resolution of $0.8 \mathrm{eV}$.

Three different types of crystal structures have been observed in STEM images of LNO along [110] zone axis. The space groups of these three phases are R-3m (layered), Fd-3m (spinel-like), and Fm-3m (rocksalt). The interesting aspect of these phases is the occupation of Li sites by Ni ions. For layered and rocksalt structures, $\mathrm{Ni}$ ions occupy - Li sites randomly, but the ratio of $\mathrm{Ni}$ ions in its original layer and in Li layer is 0.3 and 0.5 for layered and rocksalt structures respectively. So, there is a displacement of $25 \%$ to $50 \% \mathrm{Ni}$ ions from their original sites. As shown in Figure 1b, for spinel-like contrast there is an ordered variation of $\mathrm{Ni}$ ions in $\mathrm{Li}$ sites. EELS measurements of $\mathrm{O}-\mathrm{K}$ and $\mathrm{Ni}-\mathrm{L}$ edges were performed on the same region of the particles to determine the energy difference and intensity ratio of Oxygen pre and main peaks, Ni Valence, and Ni/O ratio. The Oxygen-K edge of these three types of contrast is shown in Figure 2. Surprisingly, all four of these parameters show similar values for these three contrasts with high Li content and a high Ni valence of $\sim+2.9$. These results indicate that although there is variation in STEM contrast resulting from differential movement of $\mathrm{Ni}$ ions, but chemically these phases are very similar. In all likelihood, these phases seems to be part of the large solid solution region of the phase diagram [1]. So, this work determines the equivalence of three crystal structures that are broadly 
reported as three different phases in literature, from STEM contrast and EELS measurements from the same area of the particle. Finally multislice simulations of STEM contrast are underway to precisely quantify the Ni contrast from these images. This will provide a complete picture on the nature of the phases vis-a-vis the calculated phase diagram of LNO [3].

References:

[1] Sallis S. et al, Appl. Phys. Lett. 108 (2016), p. 263902.

[2] Das H. et al, Chem. Mater. 29 (18) (2017), p. 7840.

[3] The funding for this work is provided by NECCESS, an Energy Frontier Research Center funded by the U.S. Department of Energy, Office of Science, Office of Basic Energy Sciences under Award Number DE-SC0001294. Work at the Molecular Foundry was supported by the Office of Science, Office of Basic Energy Sciences, of the U.S. Department of Energy under Contract No. DE-AC02$05 \mathrm{CH} 11231$.

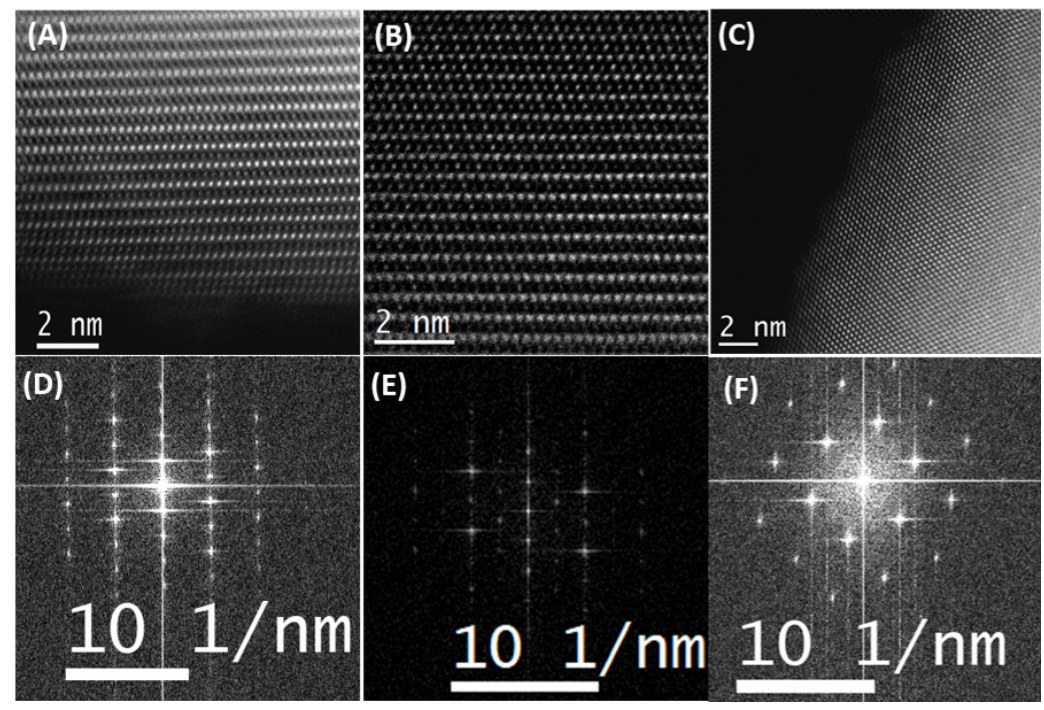

Figure 1. Variation in STEM contrast as observed along [110] zone axis in LNO held at 4.75 for 2 weeks and then discharged. The contrast and corresponding FFT are shown in pairs (A,D) layered (R$3 \mathrm{~m}),(\mathrm{B}, \mathrm{E})$ spinel-like Fd-3m and $(\mathrm{C}, \mathrm{F})$ rocksalt Fm-3m.
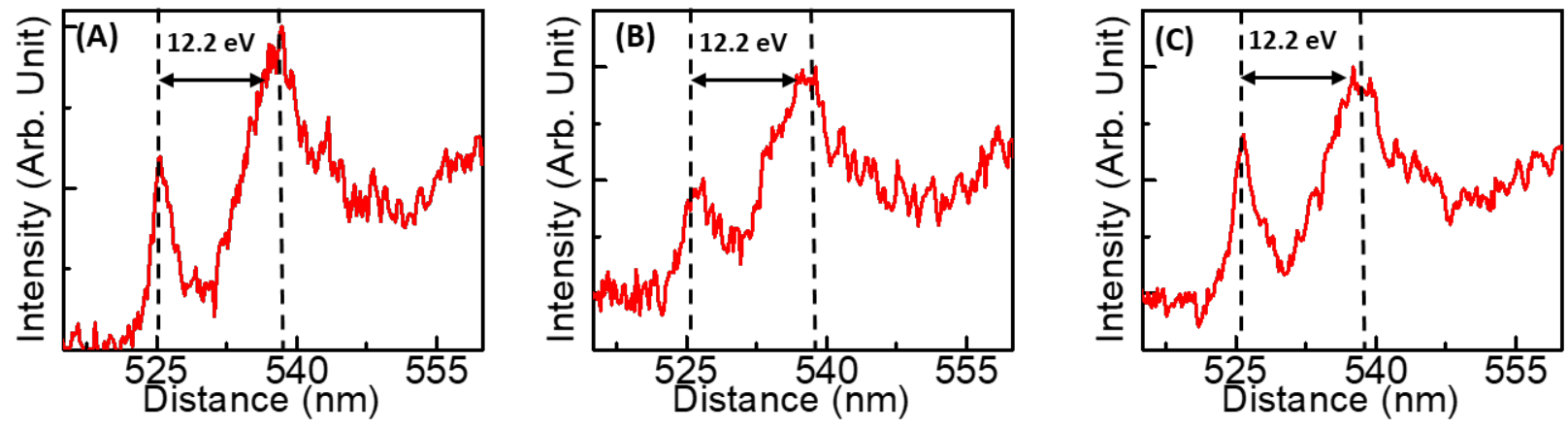

Figure 2. The Oxygen $-\mathrm{K}$ edge of as obtained from the three regions of LNO from where STEM images were obtained. (A) Layered (R-3m), (B) spinel-like Fd-3m, and (C) rocksalt Fm-3m. 